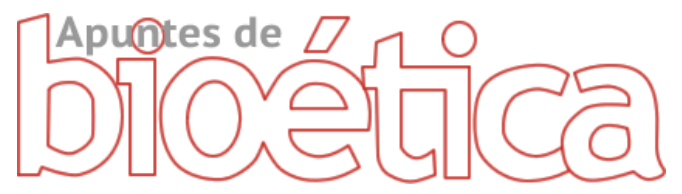

https://doi.org/10.35383/apuntes.v4i2.692

e-ISSN:2663-4910

Universidad Católica Santo Toribio de Mogrovejo

\title{
Análisis bioético y biojurídico de la normatividad sobre cuidados paliativos en Perú
}

\section{Bioethical and bio-legal analysis of the palliative care regulations in Peru}

\author{
Rosa De Jesús Sánchez-Barragán * 1, a \\ rsanchez@usat.edu.pe \\ https://orcid.org/0000-0002-7726-9775
}

Kathya Lisseth Vassallo-Cruz ${ }^{1, b}$

kvassallo@usat.edu.pe

https://orcid.org/0000-0002-6009-506X

Katherinne Jhoana Gonzales-Montenegro ${ }^{2, c}$

keymi_lebh@hotmail.com

https://orcid.org/0000-0002-1406-5433

\author{
* Autor corresponsal \\ 1, Universidad Católica Santo Toribio de \\ Mogrovejo. Chiclayo, Perú \\ 2 Independiente \\ a Doctora en Derecho \\ b Maestra en Derecho de Familia y de la Persona \\ c Estudiante de Derecho
}

\section{Fechas importantes}

Recibido: $15 / 10 / 2021$

Aceptado: 21/12/2021

Publicado online: $23 / 12 / 2021$

\section{Resumen}

Ante la situación actual y teniendo como base el contexto mundial, se brinda un análisis bioético y biojurídico de la normatividad de los cuidados paliativos en Perú. Se ha verificado que, a nivel nacional se cuenta con iniciativas relacionadas con la implementación de los cuidados paliativos, y es a partir del año 2018 que se promulga la Ley 30846: "Ley que crea el Plan Nacional de Cuidados Paliativos para Enfermedades Oncológicas y no Oncológicas"; y el Documento Técnico: "Plan Nacional de Cuidados Paliativos para Enfermedades Oncológicas y No Oncológicas en el Perú 2021-2023"; no obstante, ambos documentos resultan insuficientes para el abordaje efectivo de los cuidados paliativos, la protección del enfermo y el respeto de su dignidad en Perú.

Palabras clave: Cuidados Paliativos, dignidad humana, salud, enfermo, derecho, política. 


\begin{abstract}
Given the current situation and based on the world context, a bioethical and bio-legal analysis of the regulations of palliative care in Peru is provided. It has been verified that, at the national level, there are initiatives related to the implementation of palliative care, and it is as of 2018 that Law 30846 is enacted: "Law that creates the National Plan of Palliative Care for Oncological Diseases and not Oncological "; and the Technical Document: "National Plan of Palliative Care for Oncological and NonOncological Diseases in Peru 2021-2023"; However, both documents are insufficient for an effective approach to palliative care, the protection of the patient, and respect for their dignity in Peru.
\end{abstract}

Keywords: Palliative care, human dignity, health, sick, law, politics.

\title{
Introducción
}

La realidad peruana muestra un aumento en la expectativa de vida de la población. Al respecto, el Centro Nacional de Planeamiento Estratégico (2020) en el Documento de Trabajo denominado Perú 2050: tendencias nacionales con el impacto de la COVID 19, señaló que existe:

una tendencia al incremento en el número de personas mayores de 60 años de edad. Hacia el 2021, el país albergaría a 3,7 millones de personas mayores de 60 años de edad (11,2\% de la población total); hacia el 2030 , se situaría en 5,1 millones $(14,3 \%$ de la población total), y para el 2050, sería 8,7 millones de personas (21,8\% de la población total en el 2050) (p. 34).

Además, se espera que en el 2040 la población mayor de 60 años llegue a sobrepasar a los menores de 15 años de edad (INEI, 2020). Lo que motiva a estudiar el contexto sanitario, y concretamente la situación actual de los Cuidados Paliativos en Perú, desde el plano social y jurídico, que nos permita introducirnos a un análisis bioético y biojurídico del tema en cuestión.

Los cuidados paliativos, han sido definidos por la Organización Mundial de la Salud (OMS), como:

los cuidados apropiados para el paciente con una enfermedad avanzada y progresiva donde el control del dolor y otros síntomas, así como los aspectos psicosociales y espirituales cobran la mayor importancia. El objetivo de los cuidados paliativos es lograr la mejor calidad de vida posible para el paciente y su familia. La Medicina paliativa afirma la vida y considera el morir como un proceso normal. Los cuidados paliativos no adelantan ni retrasan la muerte, sino que constituyen un verdadero sistema de apoyo y soporte para el paciente y su familia. (Organización Panamericana de la Salud, 1998, p. 3) 
Por su parte, el Informe de la Organización Mundial de la Salud -OMS (2020), señaló que los Cuidados Paliativos son reconocidos dentro del derecho humano a la salud, debiéndose brindar servicios integrales que se encuentren dirigidos a la persona y atienda sus necesidades. En este sentido, el derecho a la salud debe abarcar aquellos mecanismos de protección y ayuda a las personas más vulnerables, siendo los cuidados paliativos uno de ellos, medio idóneo que amerita ser implementado y brindado al enfermo.

Dentro de este orden de ideas, los cuidados paliativos al ser parte del contenido del derecho a la salud, se encuentran estrechamente vinculados y alineados con el respeto de la dignidad humana, principio que se encuentra reconocido en nuestra Constitución Política del Perú, y conceptualizado por el Tribunal Constitucional Peruano (2007), máximo intérprete de la Constitución, en la sentencia del Expediente 10087-2005-PA, donde define a la dignidad de la persona humana como un valor y un principio constitucional que exige un trato digno por parte de los demás miembros de la sociedad, y prohíbe que la persona sea instrumentalizada y tratada como mero objeto del poder del Estado, siendo "parámetro fundamental de la actividad del Estado y de la sociedad, así como la fuente de los derechos fundamentales" (Fundamento 5).

Los cuidados paliativos salvaguardan esta dignidad humana a través del accionar de un equipo multidisciplinario coordinado, formado por médicos de diversas especialidades, enfermeras, psicólogos, asistentes sociales, nutricionistas, fisioterapeutas, terapeutas ocupacionales, entre otros profesionales involucrados. Citando a la OMS (1990), podemos decir que entre los objetivos más importantes de los cuidados paliativos se encuentra el "reafirmar la importancia de la vida, considerando a la muerte como un proceso normal" ( $p$. 12), debiéndose respetar la dignidad del ser humano hasta el término de su existencia.

Es fundamental que esta dignidad inviolable se pueda custodiar a través de la institución de los cuidados paliativos. El acompañamiento en la enfermedad y el proceso de morir nos recuerda que tenemos el derecho a la vida, y con ello a vivir humanamente la propia muerte, aceptando la finitud de nuestra condición humana y respetando nuestra dignidad, aún en condiciones de extrema debilidad y dependencia (Taboada, 2000). La persona en situación de enfermedad será siempre digna, porque el ser digno obedece al "ser" y no a las circunstancias accidentales que se puedan suscitar (Campos, 2020). Es a través de los 
cuidados paliativos, cuando el ser humano permanece en un estado de suma debilidad, donde aún más debe ser reconocido el valor inalienable de su dignidad humana.

El artículo tiene como objetivo mostrar el desarrollo social y jurídico de los cuidados paliativos en Perú, a fin de evidenciar si existe la necesidad de un marco de regulación biojurídica a favor del enfermo y su familia en Perú.

A fin de lograr el presente objetivo, hemos dividido el presente artículo en tres apartados: en una primera parte, se mostrará el panorama social de los cuidados paliativos en Perú; para posteriormente, en un segundo momento, describir los intentos normativos relacionados a los cuidados paliativos que se han ido gestando en Perú; concluyendo con un último apartado, donde se darán a conocer argumentos bioéticos y biojurídicos a tener en cuenta para una debida regulación de los cuidados paliativos en Perú y la importancia de efectivizar los cuidados paliativos como derecho humano a la salud del enfermo.

\section{Contexto social de los cuidados paliativos en Perú}

En Perú, según los datos plasmados por Pastrana et al. (2021) en el Atlas de Cuidados Paliativos en Latinoamérica 2020, al año 2018 son 150105 las personas que necesitan acceso a una atención en cuidados paliativos, al padecer enfermedades tales como cáncer (34\%), SIDA (19\%), demencia (17\%), enfermedades pulmonares $(3 \%)$, enfermedades cerebrovasculares (3\%), entre otras enfermedades. Sólo el 6,5\% de la referida población recibe atención paliativa.

Figura 1

Población que recibe cuidados paliativos en el Perú

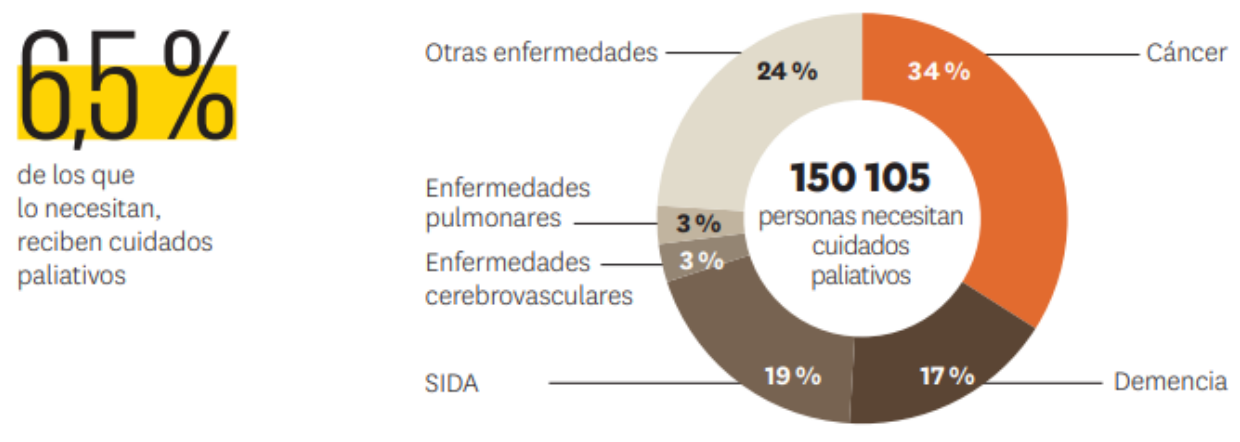

Nota. El gráfico muestra el porcentaje de la población peruana que recibe cuidados paliativos. Tomado del Atlas de Cuidados Paliativos en Latinoamérica 2a ed. (p. 51), 2020. 
Según el Documento Técnico denominado "Plan Nacional de Cuidados Paliativos para enfermedades oncológicas y no oncológicas en el Perú 2021 - 2023", de 22 regiones peruanas evaluadas entre el 2018 y 2019, los resultados arrojaron que el 67\% de la población adulta requieren de cuidados paliativos; no obstante, sólo el $0.3 \%$ se benefician con los mismos, siendo una población muy escasa aquella beneficiada con una atención de cuidados paliativos.

Por otro lado, en relación a la población pediátrica, los resultados obtenidos fueron que entre $24-27 \%$ de los niños tienen necesidad de este tratamiento, empero, la cobertura de cuidados paliativos solo oscila entre $18-20 \%$ del total. Como se aprecia en los resultados recogidos, tanto en la población adulta como en la población pediátrica, existe una atención escasa de cuidados paliativos, representando un servicio del sistema de salud con poca proyección y alcance para la población enferma.

Por otro lado, en el Atlas de Cuidados Paliativos en Latinoamérica (2020, $2^{\mathrm{a}}$ ed.), hace referencia que Perú cuenta con 19 recursos, de los cuales 10 son equipos móviles, 4 recursos de primer nivel, 4 equipos mixtos y solo 1 unidad de cuidados paliativos. De los equipos mencionados únicamente son 2 los que se encuentran destinados a cuidados paliativos pediátricos. Como podemos apreciar, se suman datos que ponen en evidencia la lenta implementación de unidades de cuidados paliativos que no responden a la demanda de aquella población que requiere este tipo de cuidados, siendo escaso su nivel de cobertura.

En el plano jurídico conviene resaltar que, en el año 2007 se produjo la aprobación de la Norma Técnica del Dolor por el Ministerio de Salud, con el fin de implementar en todos los establecimientos de salud pública y privada las llamadas unidades del dolor, algo que dista de la realidad atendiendo a las cifras antes expuestas. Posteriormente, en el año 2018, se promulgó la Ley 30846 "Ley que crea el Plan Nacional de Cuidados Paliativos para Enfermedades Oncológicas y no Oncológicas"; y en el 2021 se emitió el Documento Técnico del "Plan Nacional de Cuidados Paliativos para Enfermedades Oncológicas y No Oncológicas en el Perú 2021-2023" promulgado por Resolución Ministerial № 939-2021/MINSA, de fecha 26 de julio del 2021. 
A nivel del sector educativo peruano, cabe resaltar que no se cuenta con una acreditación oficial de la especialización en medicina paliativa, lo que sí se puede detectar en alguna de las facultades de ciencias de la salud, son asignaturas independientes de cuidados paliativos. Así tenemos que, de 26 facultades de medicina a nivel nacional solo 1 facultad cuenta con dicha asignatura; mientras que en enfermería, de las 14 escuelas sólo 3 cuentan con la asignatura de cuidados paliativos.

Sobre programas universitarios relacionados a cuidados paliativos, cabe destacar la iniciativa académica del Instituto de Bioética de la Universidad Católica Santo Toribio de Mogrovejo (USAT), que lanzó en el año 2020 un programa dirigido a profesionales que formaban parte de un equipo multidisciplinarios de cuidados paliativos o que guardaban un interés por formarse en materia paliativa, teniendo como participantes a un $60 \%$ de población internacional y un $40 \%$ de público nacional. Posteriormente se han realizado dos cursos de cuidados paliativos para personal de ESSALUD en la región Lambayeque, formando a cerca de 100 profesionales de ciencias de la salud. También otras universidades peruanas se suman con ofertas educativas, brindando maestría y diplomados sobre cuidados paliativos y tratamiento del dolor. Pese a ello todavía la apuesta y formación en cuidados paliativos es muy escasa en Perú.

\section{Contexto legal de los cuidados paliativos en Perú}

Descrito brevemente el contexto social de los cuidados paliativos en Perú, realizaremos a continuación un análisis del desarrollo legal de los cuidados paliativos en el país.

La Constitución Política del Perú, establece en el artículo 1 que "la defensa de la persona humana y su dignidad son el fin supremo de la sociedad y el Estado", y el artículo 7 reconoce que todos los peruanos tienen derecho a que su salud, medio familiar y comunidad sean protegidos, teniendo el Estado y la sociedad el deber de contribuir a su promoción y defensa. Estos dos artículos constituyen la base para la configuración de la implementación constitucional de los cuidados paliativos en Perú, que involucra a la persona enferma, a la familia y a la comunidad. 
Por otro lado, la Ley General de Salud, Ley 268462 se constituye en una norma importante a tener en cuenta. En ella se establecen los principios que guían las políticas públicas en materia de salud y regula el derecho a la protección de la salud que toda persona ostenta, siendo un derecho irrenunciable. La Ley también establece que la salud como bien jurídico, debe ser protegida al constituirse en elemento necesario para el desarrollo de la persona y su trascendencia social, siendo de interés público que el Estado establezca mecanismos para contar con una adecuada política pública de salud que proteja a los más vulnerables.

La Ley 29414, "Ley que establece los derechos de las personas usuarias de los servicios de salud", de fecha 2 octubre del 2009, la cual modifica algunos artículos de la Ley General de Salud en relación a los accesos a la salud. Cabe destacar lo siguiente cambios: establece el derecho que todo paciente tiene de acceder de forma adecuada a los servicios de salud a fin de contar con mecanismos idóneos para hacer efectivo el ejercicio de su derecho a la salud. Asimismo, reconoce el derecho que tienen los enfermos a recibir información adecuada, comprensible y oportuna sobre la enfermedad y diagnóstico, así como alternativas de tratamiento. Además, en su artículo 15.3.a. señala que el paciente debe ser atendido respetando su dignidad e intimidad, respetándose en la persona enferma el proceso natural de su muerte. Y por último, hace referencia a la necesidad de que los pacientes brinden su consentimiento informado de forma libre y voluntaria, sin que medien mecanismos que puedan viciar su voluntad.

Posteriormente, con fecha 13 de agosto del 2015 se promulgó el Decreto Supremo No 0272015-SA, por el cual se aprueba el Reglamento de la Ley 29414 "Ley que establece los derechos de las personas usuarias de los servicios de salud", en la cual se brinda alcances sobre los derechos al acceso de servicios y atención integral de salud, así como a la información y consentimiento informado que deben ser ofrecidos a la persona usuaria. En el artículo $17^{\circ}$ se hace mención al derecho que tiene todo enfermo a continuar o desistir del tratamiento, tras ser informado sobre las alternativas de tratamiento para su enfermedad. Ello evidencia que toda práctica realizada por el personal de salud debe ser tendente a buscar el bien del paciente y a informarlo debidamente sobre su enfermedad y alternativas de tratamiento, a fin de que el paciente tome una decisión razonada a la luz del referido artículo. Además, el artículo $23^{\circ}$, constituye una de las primera normas que hace referencia de forma expresa al tema de los cuidados paliativos, en ella se señala que la persona 
enferma tiene derecho a que el proceso natural de su muerte sea respetado, y que se le brinden los cuidados paliativos adecuados a su situación de enfermedad, si bien esta norma destaca la importancia y atención que se le debe dar al enfermo en estado terminal; cabe precisar que, los cuidados paliativos no debe ser aplicados sólo para aquellos enfermos en situación terminal, sino que debe evaluarse la pertinencia o no de su aplicación desde el momento que se diagnostica una enfermedad y no esperar que sea empleada únicamente en fase terminal.

Luego, un 19 de setiembre del 2018, se promulgó la Ley 30846 "Ley que crea el Plan Nacional de Cuidados Paliativos para enfermedades oncológicas y no oncológicas", la cual sólo contiene tres artículos, donde ordena el Poder Ejecutivo a través del Ministerio de Salud, la creación del Plan Nacional de Cuidados Paliativos para Enfermedades Oncológicas y No Oncológicas, y señala que dicho Plan tiene como objetivo general asegurar la inclusión de los cuidados paliativos en el Sistema Nacional de Salud. Lo llamativo de esta norma es que sólo dispone la inclusión, pero no indica la forma en que debe realizarse tal cometido.

Cabe destacar que, el 10 de marzo de 2021, Perú como estado miembro de las Naciones Unidas (ONU), ratificó la Convención Interamericana de Derechos Humanos de las personas mayores (2015), la cual en su artículo 12 obliga a los estados partes a crear un sistema integral de cuidados paliativos, además de proporcionar una definición de Cuidados Paliativos.

En esa línea, con fecha 26 de julio del 2021, el Estado Peruano aprobó por Resolución Ministerial N 939-2021/MINSA, el Documento Técnico: "Plan Nacional de Cuidados Paliativos para enfermedades Oncológicas y no Oncológicas en el Perú 2021 - 2023". El presente documento técnico, contiene lineamientos y principios emitidos por la Organización Mundial de la Salud, así mismo se menciona la situación actual de los cuidados paliativos en Perú, precisa sus antecedentes, base legal, alternativas de solución, hace un llamado a las diversas entidades para la implementación y ejecución de este Plan. El objetivo general de este Documento Técnico (2021) es: "Mejorar la respuesta del Sistema Nacional de Salud para la atención de cuidados paliativos de las personas con enfermedades oncológicas y no oncológicas, sus cuidadores, familia y comunidad, durante el periodo 2021 - 2023" (p.5). 
También se establece en dicho documento que, durante el periodo 2021-2023 se deben desarrollar diversas acciones tendentes a difundir el referido Plan a nivel local, regional y nacional; y a su vez, se encamine a la implementación de documentos y estrategias que incluyan la prestación de este tratamiento integral de cuidados paliativos en el sistema sanitario peruano.

Es importante resaltar que el presente documento técnico, en cumplimiento de lo requerido por la Convención Interamericana de Derechos Humanos de las personas mayores (2015), define a los cuidados paliativos como aquella atención ofrecida a personas que padecen alguna enfermedad severa que requiere tratamiento. Siendo los cuidados paliativos parte de la atención integral de salud que se debe brindar al paciente, su entorno y familia.

Asimismo, encontramos en este documento la clasificación que se efectúa de los cuidados paliativos. Así tenemos comprendidos a:

-Cuidados Paliativos Básicos: son aquellos cuidados brindados a los pacientes que no requieren especialistas para la atención de sus necesidades, ya que pueden ser cubiertas por el equipo de Atención Primaria. Dos tercios de los pacientes que requieren Cuidados Paliativos podrían estar en este nivel.

-Cuidados Paliativos Domiciliarios: estos cuidados están destinados a aquellos pacientes que requieren un conjunto de prestaciones y/o actividades en el domicilio, lo cuales son brindados por el sistema asistencial con la finalidad de apoyar a los pacientes, familiares y cuidadores asistiéndolos de forma organizada y programada con actividades ofrecidas por los propios establecimientos de salud. Se articula mediante el sistema de referencia y contrarreferencia, a fin de prestar un adecuado servicio de salud al usuario, de acuerdo al nivel de atención y grado de complejidad, son los establecimientos de salud del primer y/o segundo y/o tercer nivel de atención donde se evalúa su capacidad resolutiva.

- Cuidados Paliativos Especializados: Son aquellos cuidados brindados a pacientes de alta complejidad que tienen necesidades médicas mayores, los cuales deben ser abordados por especialistas de forma individualizada, en coordinación con los proveedores de los cuidados paliativos intermedios y básicos, a través del sistema de referencia y contrarreferencia. 
- Cuidados Paliativos Intermedios: Son los cuidados brindados a aquellos pacientes con enfermedades limitantes, que pueden tener exacerbaciones de dolor u otros síntomas, o distrés social o emocional, que requieran acudir a un establecimiento de salud que brinde servicio de Cuidados Paliativos en coordinación con los proveedores de los Cuidados Paliativos básicos, por medio del sistema de referencia y contrarreferencia.

De forma específica, consideramos que este último documento, es un escalón más para la inclusión de los cuidados paliativos como un derecho, el cual debe ser atendido por el sistema nacional de salud peruano. Mientras que en líneas generales, la clasificación realizada por la normatividad, demuestra la necesidad de contar con un conocimiento cada vez más profundo sobre la atención a los enfermos oncológicos o no oncológicos, a fin de poderles brindar una atención digna que los acompañe en el proceso de la enfermedad que padecen. 
A continuación, se ofrece la siguiente tabla resumen a través de la cual se ha diseñado un recorrido normativo de la evolución de los cuidados paliativos en Perú:

\section{Tabla 1}

\section{Recorrido Normativo de los Cuidados Paliativos en Perú}

\begin{tabular}{|c|c|c|}
\hline Año & $\begin{array}{c}\text { Instituto, asociación o } \\
\text { normativa }\end{array}$ & Acontecimiento importante \\
\hline 1989 & $\begin{array}{l}\text { Hospital de la Policía Nacional del } \\
\text { Perú }\end{array}$ & $\begin{array}{l}\text { Primera unidad del dolor y cuidados. } \\
\text { paliativos, gracias a la iniciativa de Raymundo } \\
\text { Cordero Lujan y María Berenguel Cook. }\end{array}$ \\
\hline 1997 & $\begin{array}{l}\text { Ley } N^{\circ} \text { 26842: Ley General de } \\
\text { Salud } \\
\text { Elaboración del Plan Nacional para el }\end{array}$ & $\begin{array}{l}\text { La protección de la salud es de interés } \\
\text { vúblico. }\end{array}$ \\
\hline 2004 & $\begin{array}{l}\text { fortalecimiento de la prevención y } \\
\text { el control del cáncer }\end{array}$ & Creación de la coalición contra el cáncer. \\
\hline 2007 & $\begin{array}{l}\text { Aprobación de la norma técnica del } \\
\text { dolor por el Ministerio de Salud }\end{array}$ & $\begin{array}{l}\text { Tratamiento del Dolor en los establecimientos } \\
\text { de salud públicos y privados. }\end{array}$ \\
\hline 2013 & $\begin{array}{l}\text { Sociedad Peruana de Cuidados } \\
\text { Paliativos (SPCP) }\end{array}$ & $\begin{array}{l}\text { Asociación sin fines de lucro que tiene como } \\
\text { misión que promueve el cuidado paliativo, a } \\
\text { través de la investigación, ética y } \\
\text { comunicación de todos los profesionales } \\
\text { involucrados en mejorar la calidad de vida de } \\
\text { los pacientes con enfermedades incurables } \\
\text { proaresivas. } \\
\text { Se aprueba el Reglamento de la Ley } 29414\end{array}$ \\
\hline 2015 & Decreto Supremo No 027-2015-SA & $\begin{array}{l}\text { Ley que establece los derechos de las } \\
\text { personas usuarias de los servicios de salud. }\end{array}$ \\
\hline 2018 & $\begin{array}{l}\text { Ley 30846: "Ley que crea el Plan } \\
\text { Nacional de Cuidados Paliativos para } \\
\text { Enfermedades Oncológicas y no } \\
\text { Oncológicas" }\end{array}$ & $\begin{array}{l}\text { Asegurar la inclusión de los cuidados paliativos } \\
\text { en el Sistema Nacional de Salud. }\end{array}$ \\
\hline 2019 & $\begin{array}{l}\text { Ley 29414: Ley que establece los } \\
\text { derechos de las personas usuarias } \\
\text { de los servicios de Salud }\end{array}$ & $\begin{array}{l}\text { Modifica algunos artículos de la Ley General } \\
\text { de Salud en relación a los accesos a la salud. }\end{array}$ \\
\hline \multirow[t]{2}{*}{2021} & $\begin{array}{l}\text { Perú ratifica la Convención } \\
\text { Interamericana sobre la protección } \\
\text { de los derechos humanos de las } \\
\text { personas mayores. }\end{array}$ & $\begin{array}{l}\text { Octavo país en ratificar la Convención, y } \\
\text { donde reconocen a los cuidados paliativos } \\
\text { dentro del derecho a la salud, como servicios } \\
\text { que deben estar disponibles y ser accesibles } \\
\text { que abarcaquen al paciente, su entorno y su } \\
\text { familia. }\end{array}$ \\
\hline & $\begin{array}{l}\text { Documento Técnico: "Plan Nacional } \\
\text { de Cuidados Paliativos para } \\
\text { Enfermedades Oncológicas y No } \\
\text { Oncológicas en el Perú } 2021 \text { - }\end{array}$ & $\begin{array}{l}\text { Planteamiento que insta a mejorar la calidad } \\
\text { de vida de los pacientes (adultos, niños y } \\
\text { neonatos). }\end{array}$ \\
\hline
\end{tabular}

Nota. En la tabla se muestra en orden cronológico las normativas peruanas vigentes al 2021, relacionada a los cuidados paliativos. Creación propia.

Como podemos apreciar, se verifica desde 1989 a la actualidad, la existencia de pequeños intentos por incorporando los cuidados paliativos en el sistema de salud peruano, pero no se cuenta con una ley específica de cuidados paliativos en Perú; por otro lado, existe muy poca formación a nivel nacional de profesionales de salud capacitados en cuidados paliativos y tratamiento del dolor. 


\section{Análisis bioético y biojurídico de los cuidados paliativos en Perú}

Abordar los cuidados paliativos desde la bioética es fundamental para todos: pacientes, familias y profesionales. En este sentido Bátiz (2021) sostiene que, el cuidado del enfermo terminal es complejo y debe recibir una atención especializada en cuidados paliativos, al verse involucrada una triple realidad (enfermo, profesionales sanitarios y familia, que deben ponerse de acuerdo por su papel importante en el proceso de morir), trayendo consigo conflictos bioéticos sobre los cuales se deben tomar decisiones apropiadas, para el bien de la persona y el respeto de su dignidad, enlazando ciencia y sabiduría práctica para atender cada caso concreto con la prudencia que se requiere.

En este sentido los cuidados paliativos son un servicio de salud que contribuye al cuidado de la persona en el proceso de morir, ayudándole a sobrellevar la enfermedad respetando su dignidad. Además, la bioética nos ayuda a pensar y a razonar con rigor, de tal manera que podamos explicar y argumentar por qué tomamos una decisión centrada en la persona y no otra. Además nos ayuda a tomar en cuenta que todo enfermo en la etapa en la que se encuentre necesita a "alguien" a su lado, éste reclama a un "otro" en cual entre un apoyo humano, médico y jurídico.

En este sentido, Cabrera et al. (2021) refiere que en el final de la vida, se debe avalar un tratamiento integral en todos los ámbitos sanitarios. De este modo, se incorporaría una mejor inspección y acompañamiento de los síntomas que cualifican a un ser humano, como multidimensional (físicos, psicológicos, sociales y espirituales). Resulta claro, que la humanización del derecho a la salud al final de la vida, se encuentra íntimamente relacionada con cuidar y respetar la dignidad inviolable de la persona hasta el culmen de su existencia (la cual, antes de ser una historia clínica es una historia de vida); por ende, recibir cuidados de atención paliativa, con competencia y humanismo (al igual que Saunders, mezclando ciencia y humanidad) no debería constituir sólo una prerrogativa, sino más bien se constituye dentro del derecho a la salud de toda persona en general y del enfermo en particular (oncológico y no oncológico). 
Todo problema bioético tiene incidencia en el derecho, convirtiéndose en problema jurídico, así tenemos que los Cuidados Paliativos se convierten también en un tema para ser abordado no sólo por la medicina sino también por la biojurídica.

Aparisi (1993) señala que:

(...) la biojurídica abordaría, asimismo, cuestiones bioéticas, pero adoptando una perspectiva distinta, la aportada por la filosofía jurídica. De este modo, la función de la biojurídica será, precisamente, la de justificar aquellos principios, no morales, sino propiamente jurídicos, en los que se apoyaría el bioderecho o la biolegislación.

En este sentido podemos decir que la biojurídica le corresponde -al igual que la filosofía del derecho- reflexionar sobre las normas vigentes, a la luz del principio de la dignidad humana y de los derechos humanos, preguntándose no por la validez de la norma, sino por lo justo de su contenido.

A continuación, haremos un breve análisis desde la perspectiva biojurídica de la normatividad peruana relacionada a los cuidados paliativos. Si bien en Perú, los cuidados paliativos surgen entre la década de los 80 y 90, con la finalidad de otorgar una respuesta al tratamiento inadecuado del sufrimiento de paciente al final de su existencia, es con el Plan Nacional del 2018 que se evidencian aspectos biojurídicos a ser tomados en cuenta en su regulación y normatividades futuras.

Una definición biojurídica, ubicable en el actual Plan Nacional, es la referida a la "medicina centrada en la persona", que se enfoca en el paciente y sus dimensiones humanas (biológica, psicológica, sociocultural y espiritual) con una visión y quehacer integrales, desde una atención clínica individual, hasta el servicio de salud pública. Aspira, por ello, a ser personalizada, entendiendo a cada paciente como fenómeno único (persona) con sus aspectos de salud negativa (enfermedad y dolencia) y de salud positiva (bienestar y desarrollo personal). Como objetivo final, propone integrar el quehacer médico, científico y humanista en la relación médico-paciente y la conexión que debe existir entre saludpoblación. 
En relación al desarrollo de la atención domiciliaria en Cuidados Paliativos en Perú, el Plan considera diferentes modelos organizativos, como son: la Asistencia Domiciliaria Geriátrica Hospital Central FAP (HCFAP); el servicio de control post hospitalario especializado (COPHOES) de EsSalud en la red de Rebagliati y Sabogal; el Programa de Atención Domiciliaria del Adulto Mayor - ADAMO (2013), que tiene convenio entre Gobierno Regional del Callao y el Fondo Intangible Solidario de Salud (FISSAL) del pliego Seguro Integral de Salud (SIS); y el programa de Atención Primaria Integral Domiciliaria Oncológica (ADI Oncológica) del Hospital Cayetano Heredia que, además de la atención ambulatoria, realiza atención domiciliaria, articulado con establecimientos de salud del primer nivel de atención de la Dirección de Redes Integradas de Salud (DIRIS) de Lima Norte; formando parte de los diferentes modelos organizativos existentes a nivel internacional, que cuentan con equipos de atención domiciliaria de Cuidados Paliativos, y que han demostrado ser coste efectivo para el sistema de salud, los cuales deberían seguirse impulsando.

Lo antes mencionado, evidencia la necesidad de establecer en nuestro ordenamiento jurídico peruano una atención primaria de salud al final de la vida, que debe ser vista como aquello que en efecto es, parte del contenido del derecho a la salud del paciente, llamada a "respetar su vida y dignidad, no causarles nunca daño, mitigar su dolor y otros síntomas con la prudencia y energía necesaria, sabiendo que se está actuando sobre un organismo particularmente vulnerable" (Bátiz, 2021, p.170). Apremia en Perú una macro - gestión sanitaria que jurídicamente tenga el deber de tratar al enfermo como un ser humano hasta el momento de su muerte, que involucra tanto al derecho como a la política.

Sobre el derecho, cabe referir que se trata de una herramienta racional al servicio de la mejor convivencia, y está llamado a proteger a la persona y de forma especial al más débil y vulnerable, recayendo en el Estado y la sociedad el deber de salvaguardar sus derechos. A decir de Hervada (2012), el derecho "es aquella cosa que, estando atribuida a un sujeto, que es su titular, es debida a este, en virtud de una deuda en sentido estricto" (p.75). Por tanto, qué es lo debido en el caso concreto: es la protección de la vida y la salud, que implica un derecho a conservarla, y ver los mecanismos adecuados en sentido los cuidados paliativos constituyen el mecanismo idóneo para cumplir con esa finalidad. En el caso materia de estudio, el enfermo es a quien se debe brindar los mecanismos de atención necesarios para acompañarlo en el proceso de su enfermedad hasta el término de su vida, 
que siempre será digna en todo momento. Asimismo, se deben orientar las políticas sanitarias de nuestro país sobre la base del principio de dignidad de la persona y el respeto de los derechos a la vida y salud. Por todo lo mencionado anteriormente, resulta necesaria la implementación de la Ley 30846, y al mismo tiempo difundir, enriquecer y ejecutar del Plan Nacional de Cuidados Paliativos.

\section{Conclusiones}

En Perú, la atención en cuidados paliativos se inicia desde 1989, a través del manejo de dolor en unidades creadas para este fin; no obstante, en estos últimos años han surgido -en el sector público y privado- algunas iniciativas que resultan ser escasas e insuficientes para atender una necesidad que desde siempre ha estado presente y que viene siendo postergada continuamente por las políticas sanitarias.

Los cuidados paliativos en Perú, de forma expresa se encuentran custodiados por la Ley $\mathrm{N}^{\circ}$ 30846, Ley que crea el Plan Nacional de Cuidados Paliativos para Enfermedades Oncológicas y no Oncológicas, y por el Documento Técnico: Plan Nacional de Cuidados Paliativos para enfermedades oncológicas y no oncológicas en el Perú 2021-2023, si bien se encuentran custodiados, su regulación constituye un tema pendiente en nuestro país.

El aporte bioético y biojurídico constituye un elemento importante para hacer efectivo el derecho humano a la salud, donde los cuidados paliativos se presentan como parte del contenido esencial del derecho y no resulta ser algo extraordinario de lo que pueda prescindir el Estado al momento de regular sus políticas públicas sanitarias. Por tanto se requiere que exista un tratamiento jurídico adecuado de los cuidados paliativos para custodiar la dignidad de todo enfermo.

A la exigencia bioética y biojurídica de brindar cuidados paliativos para resguardar la salud y dignidad de la persona enferma a la que se debe acompañar en el proceso de final de vida, se suma el aumento de las expectativas de vida de la población peruana, así como la alta demanda de la población que requiere de cuidados paliativos en Perú; sin duda, estamos 
frente a una necesidad de Cuidados Paliativos que se encuentra en constante aumento en el país y que exige ser cubierta y atendida.

\section{Referencias}

Apasiri, A. (2013). El principio de la dignidad humana como fundamento de un bioderecho global. Cuadernos de Bioética, 24 (81), 205-209. https://bit.ly/3soU2gY

Bátiz, J. (2021). Mientras llega la muerte. Reflexiones en torno al final de la vida. Madrid: Fundación San Juan de Dios.

Cabrera, M. E., Arévalo, S., \& Irigoyen, A. (2021). Cuidados Paliativos, una disciplina que humaniza la práctica médica al final de la vida: Algunos aportes desde la psicología. Apuntes De Bioética, 4(1), 122-143. https://doi.org/10.35383/apuntes.v4i1.608

Campos, P. (2020). La dignidad del enfermo en situación terminal, Apuntes De Bioética, 3 (1), 5-11. https://doi.org/10.35383/apuntes.v3i1.367

Centro Nacional de Planeamiento Estratégico (2020). Documento de Trabajo denominado Perú 2050: tendencias nacionales con el impacto de la COVID. Lima: CEPLAN. 19 https://bit.ly/3H2yECq

Constitución Política del Perú (1993).

Decreto Supremo 027-2015-SA (2015). Aprueban Reglamento de la Ley 29414, Ley que establece los derechos de las personas usuarias de los servicios de salud, 13 de agosto de 2015, Perú.

Decreto Supremo 044-2020-RE (2021). Ratificación de la Convención Interamericana de Derechos Humanos de las personas mayores, 10 de marzo de 2021, Perú.

Hervada, J. (2012). Temas de Filosofía del Derecho. Pamplona: EUNSA.

Instituto Nacional de Estadística (2020). Estado de la población peruana 2020. UNFPA. https://bit.ly/3EiqvYG

Ley 268462 (1997). Ley General de Salud, 15 de julio de 1997, Perú.

Ley 29414 (2009). Ley que establece los derechos de las personas usuarias de los servicios de salud, 2 octubre de 2009, Perú.

Ley $N^{\circ} 30846$, Ley que crea el Plan Nacional de Cuidados Paliativos para enfermedades oncológicas y no oncológicas, 24 de agosto de 2018, Perú.

Ley No 30846 (2018). Ley que crea el Plan Nacional de Cuidados Paliativos para enfermedades oncológicas y no oncológicas, 19 de setiembre de 2018, Perú.

Ministerio de Salud (2007). Norma Técnica de Salud de la Unidad Productora de Servicios Tratamiento del Dolor. NTS Nº 062-MINSA/DGSP-V.01, 06 de diciembre de 2007, Perú. 
Ministerio de Salud (2021). Documento Técnico: Plan Nacional de Cuidados Paliativos para Enfermedades Oncológicas y No Oncológicas en el Perú 2021 - 2023. Resolución Ministerial № 939-2021/MINSA, 26 de julio de 2021, Perú.

Organización Mundial de la Salud (1990). Alivio del dolor y tratamiento paliativo en cáncer. Informe de un Comité de expertos (Serie de Informes Técnicos 804). Ginebra: OMS. https://bit.ly/32rjwPH

Organización Mundial de la Salud (2020). Cuidados Paliativos. Datos y Cifras. 20 de agosto 2020. https://bit.ly/3FlcOJU

Organización Panamericana de la Salud (1998). Cuidados Paliativos. Guías para el Manejo Clínico. OPS. https://bit.ly/3yLTvXH

Pastrana, T.; De Lima, L.; Sánchez-Cárdenas, M.; Van Steijn, D.; Garralda, E.; Pons, JJ. y Centeno, C. (2021). Atlas de Cuidados Paliativos en Latinoamérica 2020. ( $2^{\mathrm{a}}$ ed.). Houston: IAHPC Press.

Taboada, P. (2000). El derecho a morir con dignidad. Acta bioethica, 6(1), 89-101. https://dx.doi.org/10.4067/S1726-569X2000000100007

Tribunal Constitucional Peruano (2007). Expediente 10087-2005-PA, 18 de diciembre de 2007.

\section{Cómo citar este trabajo}

Sánchez Barragán, R. D. J., Vassallo Cruz, K. L., \& Gonzales Montenegro, K. J. (2021). Análisis bioético y biojurídico de la normatividad sobre cuidados paliativos en Perú. Apuntes De Bioética, 4(2), 124140. https://doi.org/10.35383/apuntes.v4i2.692

\section{Financiación}

El presente artículo no cuenta con financiación específica de agencias de financiamiento en los sectores público o privado para su desarrollo y/o publicación.

\section{Conflicto de interés}

Los autores del artículo declaran no tener ningún conflicto de intereses en su realización.

(C) Los autores. Este artículo es publicado por la Revista Apuntes de Bioética del Instituto de Bioética, Universidad Católica Santo Toribio de Mogrovejo. 